九州大学学術情報リポジトリ

Kyushu University Institutional Repository

Scaling limits for the system of semirelativistic particles coupled to a scalar bose field

Takaesu, Toshimitsu

Faculty of Mathematics, Kyushu University

http://hdl. hand le. net/2324/16962

出版情報: MI Preprint Series. MI 2010-18, 2010-04-16. 九州大学大学院数理学研究院 バージョン：

権利関係: 


\section{Preprint Series}

Kyushu University

The Global COE Program

Math-for-Industry Education \& Research Hub

\section{Scaling limits for the system of semi-relativistic particles coupled to a scalar bose field}

\section{Toshimitsu Takaesu}

MI 2010-18

( Received April 16, 2010 )

Faculty of Mathematics

Kyushu University

Fukuoka, JAPAN 


\title{
Scaling Limits for the System of Semi-Relativistic Particles Coupled to a Scalar Bose Field
}

\author{
Toshimitsu TAKAESU
}

Faculty of Mathematics, Kyushu University, Fukuoka, 812-8581, Japan

\begin{abstract}
In this paper the Hamiltonian for the system of semi-relativistic particles interacting with a scalar bose field is investigated. A scaled total Hamiltonian of the system is defined and its scaling limit is considered. Then the semi-relativistic Schrödinger operator with an effective potential is derived.
\end{abstract}

Mathematics Subject Classification $2010: 81 \mathrm{Q} 10,62 \mathrm{M} 15$.

key words : Spectral analysis, Relativistic Schrödinger operator, Quantum field theory.

\section{Introduction}

In this paper we consider the Hamiltonian of the system of $N$ particles linearly coupled to a scalar bose field. We assume that particles obey the semi-relativistic Schrödinger operator

$$
H_{\mathrm{p}}=\sum_{j=1}^{N} \sqrt{-\triangle_{j}+M^{2}}
$$

where $M>0$ is a rest mass. There has been many results on the spectral properties of $H_{\mathrm{p}}$. Refer to e.g. $[21,6,3,4,5,20]$. The free Hamiltonian $H_{\mathrm{b}}$ of the scalar bose field is defined by the second quantization of the multiplication operator $\omega$, which is formally expressed by

$$
H_{\mathrm{b}}=\int_{\mathbf{R}^{d}} \omega(\mathbf{k}) a^{*}(\mathbf{k}) a(\mathbf{k}) d \mathbf{k}
$$

The state space of the interacting system is defined by $\mathcal{H}=L^{2}\left(\mathbf{R}_{\mathbf{x}}^{d N}\right) \otimes \mathcal{F}_{\mathbf{b}}\left(L^{2}\left(\mathbf{R}_{\mathbf{k}}^{d}\right)\right)$ where $\mathcal{F}_{\mathbf{b}}\left(L^{2}\left(\mathbf{R}_{\mathbf{k}}^{d}\right)\right)$ is the boson Fock space on $L^{2}\left(\mathbf{R}_{\mathbf{k}}^{d}\right)$. The total Hamiltonian is given by

$$
H=H_{\mathrm{p}} \otimes I+I \otimes H_{\mathrm{b}}+\kappa H_{\mathrm{I}}, \quad \kappa \in \mathbf{R} .
$$

Here the interaction $H_{\mathrm{I}}$ is denoted by formally

$$
H_{\mathrm{I}}=\sum_{j=1}^{N} \frac{1}{\sqrt{2}} \int_{\mathbf{R}^{d}}\left(\overline{f_{\mathbf{x}_{\mathbf{j}}}(\mathbf{k})} \otimes a(\mathbf{k})+f_{\mathbf{x}_{\mathbf{j}}}(\mathbf{k}) \otimes a^{*}(\mathbf{k})\right),
$$

where $f_{\mathbf{x}}$ is an multiplication operator on $L^{2}\left(\mathbf{R}_{\mathbf{x}}^{d}\right)$. 
We consider the scaled Hamiltonian

$$
H(\Lambda)=H_{\mathrm{p}} \otimes I+\Lambda^{2} I \otimes H_{\mathrm{b}}+\kappa \Lambda H_{\mathrm{I}}, \quad \Lambda>0 .
$$

We investigate the asymptotic behavior of $H(\Lambda)$ as $\Lambda \rightarrow \infty$. The unitary evolution $e^{-i t H(\Lambda)}$ generated by $H(\Lambda)$ is given by

$$
e^{-i t H(\Lambda)}=e^{-i \Lambda^{2} t\left(\sum_{j=1}^{N} \sqrt{\left(\frac{\hat{\mathbf{p}}_{j}}{\Lambda^{2}}\right)^{2}+\left(\frac{M}{\Lambda^{2}}\right)^{2}}+H_{\mathrm{b}}+\left(\frac{\kappa}{\Lambda}\right) H_{\mathrm{I}}\right)} .
$$

Here $\Lambda^{2} t$ denotes the scaled time, $\Lambda^{-2} \hat{\mathbf{p}}$ the scaled momentum for $\hat{\mathbf{p}}=-i \nabla, \Lambda^{-2} M$ the scaled mass, and $\Lambda^{-1} \kappa$ the scaled coupling constant. As far as we know scaling limits of the Hamiltonians of the form (2) is initiated by E. B. Davies [2], where $H(\Lambda)$ with semi-relativistic Schrödinger operator replaced by a standard Schrödinger operator is considered and a Schödinger operator with an effective potential is derived as $\Lambda \rightarrow \infty$. This model is called the Nelson model, and our result can be regarded as a semirelativistic version of [2]. In [1], a general theory of scaling limits is established and it is applied to scaling limits of a spin-boson model and non-relativistic QED models. In [10], by removing ultraviolet cutoffs and taking a scaling limit of the Nelson model simultaneously, a Schrödinger operator with the Yukawa potential or the Coulomb potential is derived. Refer to see also $[8,17,18,16,19]$.

In the main theorem, it is shown that for $z \in \mathbf{Z} \backslash \mathbf{R}$,

$$
s-\lim _{\Lambda \rightarrow \infty}(H(\Lambda)-z)^{-1}=\left(\sum_{j=1}^{N} \sqrt{-\triangle_{j}+M^{2}}+V_{\mathrm{eff}}\left(\mathbf{x}_{1}, \cdots, \mathbf{x}_{N}\right)-z\right)^{-1} P_{\Omega_{\mathrm{b}}},
$$

where

$$
V_{\mathrm{eff}}\left(\mathbf{x}_{1}, \cdots, \mathbf{x}_{N}\right)=-\frac{\kappa^{2}}{4} \sum_{j, l} \int_{\mathbf{R}^{d}} \frac{\overline{f_{\mathbf{x}_{j}}(\mathbf{k})} f_{\mathbf{x}_{l}}(\mathbf{k})+\overline{f_{\mathbf{x}_{l}}(\mathbf{k})} f_{\mathbf{x}_{j}}(\mathbf{k})}{\omega(\mathbf{k})} d \mathbf{k},
$$

and $P_{\Omega_{\mathrm{b}}}$ is the projection onto the closed subspace spanned by the Fock vacuum $\Omega_{\mathrm{b}}$ of the bose field.

For the strategy of the proof of the main theorem, we use a unitary transformation, called the dressing transformation. Then we apply the general theory investigated in [1] to the unitary transformed Hamiltonian $U(\Lambda)^{-1} H(\Lambda) U(\Lambda)$, and the consider the asymptotic behavior of $U(\Lambda)^{-1} H(\Lambda) U(\Lambda)$ as $\Lambda \rightarrow \infty$.

This paper is organized as follows. In Section 2, the theory of boson Fock space is described. Then the total state space and the total Hamiltonian is defined, and the main results are stated. In Section 3, the proof of the main theorem is given. 


\section{Main Results}

\subsection{Boson Fock Spaces}

In this subsection we give the mathematically rigorous definition of the bose field. The state space of the bose field is given by the boson Fock space $\mathcal{F}_{\mathrm{b}}\left(L^{2}\left(\mathbf{R}^{d}\right)\right)=\oplus_{n=0}^{\infty}\left(\otimes_{\mathrm{s}}^{n} L^{2}\left(\mathbf{R}^{d}\right)\right)$, where $\otimes_{\mathrm{s}}^{n} L^{2}\left(\mathbf{R}^{d}\right)$ denotes the $n$-fold symmetric tenser product of $L^{2}\left(\mathbf{R}^{d}\right)$ with $\otimes_{s}^{0} L^{2}\left(\mathbf{R}^{d}\right):=\mathbf{C}$. The Fock vacuum is defined by $\Omega_{\mathrm{b}}=\{1,0,0, \cdots\} \in \mathcal{F}_{\mathrm{b}}\left(L^{2}\left(\mathbf{R}^{d}\right)\right)$. The finite particle subspace $\mathcal{F}_{\mathrm{b}}(\mathcal{D})$ on the subspace $\mathcal{D} \subset L^{2}\left(\mathbf{R}^{d}\right)$ is defined by the set of $\Psi=\left\{\Psi^{(n)}\right\}_{n=0}^{\infty}$ satisfying that $\Psi^{(n)} \in \otimes_{\mathrm{s}}^{n} \mathcal{D}, n \geq 0$, and $\Psi^{\left(n^{\prime}\right)}=0$ for all $n^{\prime}>N$ with some $N \geq 0$. Let $a(\xi), \xi \in L^{2}\left(\mathbf{R}^{3}\right)$, and $a^{*}(\eta), \eta \in L^{2}\left(\mathbf{R}^{d}\right)$, be the annihilation operator and the creation operator on $\mathcal{F}_{\mathrm{b}}\left(L^{2}\left(\mathbf{R}^{d}\right)\right)$, respectively. Then they satisfy the canonical commutation relations on $\mathcal{F}_{\mathrm{b}}^{\mathrm{fin}}\left(L^{2}\left(\mathbf{R}^{d}\right)\right)$ :

$$
\left[a(\xi), a^{*}(\eta)\right]=(\xi, \eta), \quad[a(\xi), a(\eta)]=\left[a^{*}(\xi), a^{*}(\eta)\right]=0 .
$$

Let $S$ be a self-adjoint operator on $L^{2}\left(\mathbf{R}^{d}\right)$. The second quantization of $S$ is defined by

$$
d \Gamma(S)=\bigoplus_{n=0}^{\infty}\left(\sum_{j=1}^{n}(I \otimes \cdots I \otimes \underbrace{S}_{j t h} \otimes I \cdots \otimes I)\right),
$$

For $\eta \in \mathcal{D}\left(S^{-1 / 2}\right)$, it is seen that $a(\eta)$ and $a^{*}(\eta)$ are relatively bounded with respect to $d \Gamma(S)^{1 / 2}$ with the bound

$$
\begin{array}{lr}
\|a(\eta) \Psi\| \leq\left\|S^{-1 / 2} \eta\right\|\left\|d \Gamma(S)^{1 / 2} \Psi\right\|, & \Psi \in \mathcal{D}\left(d \Gamma(S)^{1 / 2}\right), \\
\left\|a^{*}(\eta) \Psi\right\| \leq\left\|S^{-1 / 2} \eta\right\|\left\|d \Gamma(S)^{1 / 2} \Psi\right\|+\|\eta\|\|\Psi\|, & \Psi \in \mathcal{D}\left(d \Gamma(S)^{1 / 2}\right) .
\end{array}
$$

The field operator and its conjugate operator are defined by

$$
\phi(\xi)=\frac{1}{\sqrt{2}}\left(a(\xi)+a^{*}(\xi)\right), \quad \Pi(\eta)=\frac{i}{\sqrt{2}}\left(-a(\eta)+a^{*}(\eta)\right) .
$$

\subsection{Main Theorem}

In this subsection we define the total Hamiltonian and state the main results. The state space of the system for the $N$-particles coupled to bose field is defined by

$$
\mathcal{H}=L^{2}\left(\mathbf{R}_{\mathbf{x}}^{d N}\right) \otimes \mathcal{F}_{\mathbf{b}}\left(L^{2}\left(\mathbf{R}_{\mathbf{k}}^{d}\right)\right)
$$

The free Hamiltonian of particles and the bose field are defined by

$$
H_{\mathrm{p}}=\sum_{j=1}^{N} \sqrt{-\triangle_{j}+M^{2}}, \quad H_{\mathrm{b}}=d \Gamma_{\mathrm{b}}(\omega),
$$

where $M>0$ is a rest mass and $\omega$ denotes the multiplication operator by the function $\omega(\mathbf{k})$, which describes the energy of the boson with momentum $\mathbf{k}$. We assume the following condition :

(A.1) $\omega$ is non-negative. 
The interaction $H_{\mathrm{I}}$ is defined by

$$
H_{\mathrm{I}}=\sum_{j=1}^{N} \phi\left(f_{\mathbf{x}_{j}}\right)
$$

where $f_{\mathbf{x}}$ is the multiplication operator satisfying the following condition :

(A.2)

$$
\sup _{\mathbf{x} \in \mathbf{R}^{d}} \int_{\mathbf{R}^{d}}\left|f_{\mathbf{X}}(\mathbf{k})\right|^{2} d \mathbf{k}<\infty, \quad \text { and } \quad \sup _{\mathbf{x} \in \mathbf{R}^{d}} \int_{\mathbf{R}^{d}} \frac{\left|f_{\mathbf{X}}(\mathbf{k})\right|^{2}}{\omega(\mathbf{k})} d \mathbf{k}<\infty
$$

The total Hamiltonian of this system is given by

$$
H=H_{0}+\kappa H_{\mathrm{I}},
$$

where $H_{0}=H_{\mathrm{p}} \otimes I+I \otimes H_{\mathrm{b}}$. By (4), (5), and the assumption (A.2), it is seen that the $H_{\mathrm{I}}$ is relatively bounded with respect to $I \otimes H_{\mathrm{b}}^{1 / 2}$. Hence $H_{\mathrm{I}}$ is relatively bounded with respect to $H_{0}$ with infinitely small bound. Then the Kato-Rellich theorem shows that $H$ is self-adjoint and essentially self-adjoint any core of $H_{0}$. Then in particular, $H$ is essentially self-adjoint on $\mathcal{D}_{0}=C_{0}^{\infty}\left(\mathbf{R}^{d N}\right) \hat{\otimes} \mathcal{F}_{\mathrm{b}}^{\text {fin }}(\mathcal{D}(\omega))$, where $\hat{\otimes}$ denotes the algebraic tensor product.

Let us introduce the scaled total Hamiltonian

$$
H(\Lambda)=H_{0}(\Lambda)+\kappa \Lambda H_{\mathrm{I}},
$$

where $H_{0}(\Lambda)=H_{\mathrm{p}} \otimes I+\Lambda^{2} I \otimes H_{\mathrm{b}}$. We introduce an additional assumption on the interaction.

(A.3) $\sup _{\mathbf{x} \in \mathbf{R}^{d}} \int_{\mathbf{R}^{d}} \frac{\left|f_{\mathbf{x}}(\mathbf{k})\right|^{2}}{\omega(\mathbf{k})^{2}} d \mathbf{k}<\infty, \quad$ and $\quad \sup _{\mathbf{x} \in \mathbf{R}^{d}} \int_{\mathbf{R}^{d}} \frac{\left|f_{\mathbf{x}}(\mathbf{k})\right|^{2}}{\omega(\mathbf{k})^{3}} d \mathbf{k}<\infty$.

(A.4) $\sup _{\mathbf{x} \in \mathbf{R}^{d}} \int_{\mathbf{R}^{d}} \frac{\left|\partial_{x^{v}} f_{\mathbf{x}}(\mathbf{k})\right|^{2}}{\omega(\mathbf{k})^{2}} d \mathbf{k}<\infty, \sup _{\mathbf{x} \in \mathbf{R}^{d}} \int_{\mathbf{R}^{d}} \frac{\left|\triangle f_{\mathbf{x}}(\mathbf{k})\right|^{2}}{\omega(\mathbf{k})^{2}} d \mathbf{k}<\infty$ and $\left(\frac{\partial_{x^{v}} f_{\mathbf{x}}}{\omega}, \frac{f_{\mathbf{y}}}{\omega}\right) \in \mathbf{R}, \quad \mathbf{x}, \mathbf{y} \in \mathbf{R}^{d}$.

Under the condition $\left(\frac{\partial_{x^{v}} f_{\mathbf{x}}}{\omega}, \frac{f_{\mathbf{y}}}{\omega}\right) \in \mathbf{R}$ in (A.4), it follows that $\left[\Pi\left(\frac{\partial_{x} v f_{\mathbf{x}}}{\omega}\right), \Pi\left(\frac{f_{\mathbf{y}}}{\omega}\right)\right]=0, \mathbf{x}, \mathbf{y} \in \mathbf{R}^{d}$.

Remark 2.1 Let us define that $f_{\mathbf{x}}(\mathbf{k})=\frac{\chi_{R}(|\mathbf{k}|)}{\sqrt{\omega(\mathbf{k})}} e^{-i \mathbf{k} \cdot \mathbf{x}}$ with $\omega(\mathbf{k})=\omega(-\mathbf{k})$. Here $\chi_{R}$ denotes the characteristic function on $[0, R)$. Then the conditions (A.1)-(A.4) are satisfied, and the interaction $H_{I}$ is formally expressed by

$$
H_{I}=\sum_{j=1}^{N} \int_{\mathbf{R}^{d}} \frac{\chi_{R}(|\mathbf{k}|)}{\sqrt{2 \omega(\mathbf{k})}}\left(a(\mathbf{k}) e^{i \mathbf{k} \cdot \mathbf{x}_{j}}+a^{*}(\mathbf{k}) e^{-i \mathbf{k} \cdot \mathbf{x}_{j}}\right) d \mathbf{k} .
$$

The main theorem in this paper is as follows 
Theorem 2.1 Assume (A.1)-(A.4). Then for $z \in \mathbf{C} \backslash \mathbf{R}$ it follows that

$$
s-\lim _{\Lambda \rightarrow \infty}(H(\Lambda)-z)^{-1}=\left(H_{p}+V_{e f f}\left(\mathbf{x}_{1}, \cdots, \mathbf{x}_{n}\right)-z\right) \otimes P_{\Omega_{b}},
$$

where

$$
V_{\text {eff }}\left(\mathbf{x}_{1}, \cdots, \mathbf{x}_{n}\right)=-\frac{\kappa^{2}}{4} \sum_{j, l} \int_{\mathbf{R}^{d}} \frac{\overline{{\mathbf{x}_{j}}_{j}(\mathbf{k})} f_{\mathbf{x}_{l}}(\mathbf{k})+\overline{f_{\mathbf{x}_{l}}(\mathbf{k})} f_{\mathbf{x}_{j}}(\mathbf{k})}{\omega(\mathbf{k})} d \mathbf{k},
$$

and $P_{\Omega_{b}}$ is the projection onto the closed subspace spanned by the Fock vacuum $\Omega_{b}$.

Remark 2.2 When $f_{\mathbf{x}}(\mathbf{k})=\frac{\chi_{R}(|\mathbf{k}|)}{\sqrt{\omega(\mathbf{k})}} e^{-i \mathbf{k} \cdot \mathbf{x}}$ with $\omega(\mathbf{k})=\omega(-\mathbf{k})$, the effective potential is given by

$$
V_{e f f}\left(\mathbf{x}_{1}, \cdots, \mathbf{x}_{n}\right)=-\frac{\kappa^{2}}{2} \sum_{j, l} \int_{\mathbf{R}^{d}} \frac{\left|\chi_{R}(\mathbf{k})\right|^{2}}{\omega(\mathbf{k})^{2}} e^{-i \mathbf{k} \cdot\left(\mathbf{x}_{j}-\mathbf{x}_{l}\right)} d \mathbf{k},
$$

By using the norm convergence theorem considered in ([18] ; Lemma 2.7), the the next corollary follows.

Corollary 2.2 Assume (A.1)-(A.4). Then for $z \in \mathbf{C} \backslash \mathbf{R}$ it follows that

$$
s-\lim _{\Lambda \rightarrow \infty} e^{-i t H(\Lambda)}\left(I \otimes P_{\Omega_{b}}\right)=e^{-i t\left(H_{p}+V_{e f f}\left(\mathbf{x}_{1}, \cdots, \mathbf{x}_{n}\right)\right)} \otimes P_{\Omega_{b}} .
$$

\section{Proof of Main Theorem}

The outline of the proof of Theorem 2.1 is as follows. A unitary transformation $U(\Lambda)$, called the dressing transformation, is defined and we consider the unitarily transformed Hamiltonian $U(\Lambda)^{-1} H(\Lambda) U(\Lambda)$. Then we apply the general theory on scaling limits in [1] to $U(\Lambda)^{-1} H(\Lambda) U(\Lambda)$.

Under the condition (A.3), the following unitary operator can be defined :

$$
U(\Lambda)=e^{i\left(\frac{\kappa}{\Lambda}\right) \sum_{j=1}^{N} \Pi\left(\frac{f \mathbf{x}_{j}}{\omega}\right)} .
$$

It is seen that on the finite particle subspace

$$
\begin{aligned}
& {\left[\Pi(\xi), H_{\mathrm{b}}\right]=-i \phi(\omega \xi), \quad \xi \in \mathcal{D}(\omega),} \\
& {[\Pi(\xi), \phi(\eta)]=\frac{-i}{2}((\xi, \eta)+(\eta, \xi)), \quad \xi, \eta \in L^{2}\left(\mathbf{R}^{3}\right) .}
\end{aligned}
$$

By (6) and (7), we have

$$
U(\Lambda)^{-1} H(\Lambda) U(\Lambda)=H_{0}(\Lambda)+K(\Lambda)
$$

where

$$
K(\Lambda)=U(\Lambda)^{-1}\left(H_{\mathrm{p}} \otimes I\right) U(\Lambda)-H_{\mathrm{p}} \otimes I+V_{\mathrm{eff}}\left(\mathbf{x}_{1}, \cdots, \mathbf{x}_{N}\right)
$$

Now we apply the general theory on scaling limits investigated in [1]. Let us set the total Hilbert space by $Z=X \otimes Y$. Let $A$ and $B$ be non-negative self-adjoint operators on $X$ and $\mathcal{Y}$, respectively. Here we assume that ker $B \neq\{0\}$. We consider a family of symmetric operators $\{C(\Lambda)\}_{\Lambda>0}$ satisfying the conditions : 
(S.1) For all $\varepsilon>0$ there exists a constant $\Lambda(\varepsilon)>0$ such that for all $\Lambda>\Lambda(\varepsilon)$, $\mathcal{D}(A \otimes I) \cap \mathcal{D}(I \otimes B) \subset \mathcal{D}(C(\Lambda))$, and there exists $b(\varepsilon) \geq 0$ such that

$$
\|C(\Lambda) \Phi\| \leq \varepsilon\|(A \otimes I+\Lambda I \otimes B) \Phi\|+b(\varepsilon)\|\Phi\| .
$$

(S.2) There exists a symmetric operator $C$ on $\mathcal{Z}$ such that $\mathcal{D} \otimes \operatorname{ker} B \subset \mathcal{D}(C)$ and for all $z \in \mathbf{C} \backslash \mathbf{R})$,

$$
s-\lim _{\Lambda \rightarrow \infty} C(\Lambda)(A \otimes I+\Lambda I \otimes B-z)=C(A-z)^{-1} \otimes P_{B},
$$

where $P_{B}$ is the orthogonal projection from $y$ onto $\operatorname{ker} B$.

Proposition A ([1] ; Theorem 2.1) Assume (S.1) and (S.2). Then (i)-(iii) follows.

(i) There exists $\Lambda_{0} \geq 0$ such that for all $\Lambda>\Lambda_{0}$,

$$
X(\Lambda)=A \otimes I+\Lambda I \otimes B+C(\Lambda)
$$

is self-adjoint on $\mathcal{D}(A \otimes I) \cap \mathcal{D}(I \otimes B)$ and uniformly bounded from below for $\Lambda$, furthermore $X(\Lambda)$ is essentially self-adjoint on any core of $A \otimes I+I \otimes B$.

(ii) Let $X=A \otimes I+\left(I \otimes P_{B}\right) C\left(I \otimes P_{B}\right)$. Then $X$ is self-adjoint on $\mathcal{D}(A \otimes I)$ and bounded from below, and essentially self-adjoint on any core of $A \otimes I$.

(iii) Let $z \in \bigcap_{\Lambda \geq \Lambda_{0}} \rho(X(\Lambda)) \cap \rho(X)$, where $\rho(\mathcal{O})$ denotes the resolvent set of an operator $\mathcal{O}$. Then

$$
s-\lim _{\Lambda \rightarrow \infty}(X(\Lambda)-z)^{-1}=(X-z)^{-1}\left(I \otimes P_{B}\right) .
$$

Now we consider $H(\Lambda)$ again. What we have to prove is that $H(\Lambda)$ satisfies the condition (S.1) and (S.2) by applying $H_{0}(\Lambda)$ to $A \otimes I+\Lambda I \otimes B$ and $K(\Lambda)$ to $C(\Lambda)$. First let us consider the term $U(\Lambda)^{-1}\left(H_{\mathrm{p}} \otimes I\right) U(\Lambda)$ in $(9)$. Let us set $\hat{\mathbf{p}}=\left(\hat{p}^{1}, \cdots, \hat{p}^{d}\right)=\left(-i \frac{\partial}{\partial x^{1}}, \cdots,-i \frac{\partial}{\partial x^{d}}\right)$. Then by the spectral decomposition theorem,

$$
U(\Lambda)^{-1}\left(H_{\mathrm{p}} \otimes I\right) U(\Lambda)=\sum_{j=1}^{N} \sqrt{\left(U(\Lambda)^{-1}\left(\mathbf{p}_{j} \otimes I\right) U(\Lambda)\right)^{2}+M^{2}},
$$

follows. We see that

$$
\left[\Pi\left(f_{\mathbf{x}}\right), \hat{p}^{v}\right]=i \Pi\left(\partial_{x^{v}} f_{\mathbf{x}}\right) .
$$

Then by (A.4), it follows that for $\Psi \in \mathcal{D}_{0}$,

$$
\left(U(\Lambda)^{-1}\left(\mathbf{p}_{j} \otimes I\right) U(\Lambda)\right)^{2} \Psi=\left(\sum_{v=1}^{d}\left(\hat{p}_{j}^{v} \otimes I+\left(\frac{\kappa}{\Lambda}\right) \Pi\left(\frac{\partial_{x_{j}^{v}} f_{\mathbf{x}_{j}}}{\omega}\right)\right)^{2}+M^{2}\right) \Psi
$$

Then we have

$$
\left(U(\Lambda)^{-1}\left(\mathbf{p}_{j} \otimes I\right) U(\Lambda)\right)^{2}=\overline{\left(\sum_{v=1}^{d}\left(\hat{p}_{j}^{v} \otimes I+\left(\frac{\kappa}{\Lambda}\right) \Pi\left(\frac{\partial_{x_{j}^{v}} f_{\mathbf{x}_{j}}}{\omega}\right)\right)^{2}+M^{2}\right)_{i \mathcal{D}_{0}},}
$$


where $\bar{Y}$ denotes the closure of the operator $Y$. Here we abbreviate as

$$
\begin{aligned}
& \Pi\left(\frac{\nabla f_{\mathbf{x}}}{\omega}\right) \cdot(\hat{\mathbf{p}} \otimes I)=\sum_{v=1}^{d} \Pi\left(\frac{\partial_{x^{v}} f_{\mathbf{x}}}{\omega}\right)\left(\hat{p}^{v} \otimes I\right) \\
& \Pi\left(\frac{\nabla f_{\mathbf{x}}}{\omega}\right) \cdot \Pi\left(\frac{\nabla f_{\mathbf{x}}}{\omega}\right)=\sum_{v=1}^{d} \Pi\left(\frac{\partial_{x^{v}} f_{\mathbf{x}}}{\omega}\right) \phi\left(\frac{\partial_{x^{v}} f_{\mathbf{x}}}{\omega}\right) .
\end{aligned}
$$

Then we see that

$$
U(\Lambda)^{-1}\left(H_{\mathrm{p}} \otimes I\right) U(\Lambda)=\sum_{j=1}^{N}\left(\overline{\left(-\triangle_{j} \otimes I+Q_{j}(\Lambda)+M^{2}\right)_{\left\lceil\mathcal{D}_{0}\right.}}\right)^{1 / 2},
$$

where

$$
Q_{j}(\Lambda)=\left(\frac{\kappa}{\Lambda}\right)\left(2 \Pi\left(\frac{\nabla f_{\mathbf{x}_{j}}}{\omega}\right) \cdot\left(\hat{\mathbf{p}_{j}} \otimes I\right)-i \Pi\left(\frac{\triangle f_{\mathbf{x}}}{\omega}\right)\right)+\left(\frac{\kappa}{\Lambda}\right)^{2} \Pi\left(\frac{\nabla f_{\mathbf{x}_{j}}}{\omega}\right) \cdot \Pi\left(\frac{\nabla f_{\mathbf{x}_{j}}}{\omega}\right)
$$

Proposition 3.1 Assume (A.1)-(A.4). Then for $\varepsilon>0$, there exists $\Lambda(\varepsilon) \geq 0$ such that for all $\Lambda>\Lambda(\varepsilon)$,

$$
\left\|U(\Lambda)^{-1}\left(H_{p} \otimes I\right) U(\Lambda) \Psi-\left(H_{p} \otimes I\right) \Psi\right\| \leq \varepsilon\left\|H_{0}(\Lambda) \Psi\right\|+b(\varepsilon)\|\Psi\|
$$

where $b(\varepsilon)$ is a constant independent of $\Lambda \geq \Lambda(\varepsilon)$.

Before proving Proposition 3.1, we show the following lemma.

Lemma 3.2 For $\lambda>0$ and $\delta \in\left(0, \frac{1}{10}\right)$, there exists $M_{v}(\delta), v=1, \cdots, d$, such that

$$
\left\|\hat{p}^{v}\left(-\triangle+M^{2}+\lambda\right)^{-1}\left(\sqrt{-\triangle+M^{2}}+1\right)^{-1 / 2}\right\| \leq \frac{1}{\lambda^{\frac{1}{2}+\delta}} M_{v}(\delta)
$$

(Proof) For $\mathbf{p}=\left(p^{1}, \cdots, p^{d}\right) \in \mathbf{R}^{d}, v=1, \cdots, d$, we see that

$$
\left|p^{v}\left(\mathbf{p}^{2}+M^{2}+\lambda\right)^{-1}\left(\sqrt{\mathbf{p}^{2}+M^{2}}+1\right)^{-1 / 2}\right|=\frac{1}{\lambda^{\frac{1}{2}+\delta}}\left|\lambda^{\frac{1}{2}+\delta} p^{v}\right|\left(\mathbf{p}^{2}+M^{2}+\lambda\right)^{-1}\left(\sqrt{\mathbf{p}^{2}+M^{2}}+1\right)^{-1 / 2} .
$$

We shall show that

$$
\sup _{\lambda>0, \mathbf{p} \in \mathbf{R}^{d}}\left|\lambda^{\frac{1}{2}+\delta} p^{v}\right|\left(\mathbf{p}^{2}+M^{2}+\lambda\right)^{-1}\left(\sqrt{\mathbf{p}^{2}+M^{2}}+1\right)^{-1 / 2}<\infty,
$$

and hence (15) follows from the spectral decomposition theorem. The Young's inequality shows that for $q>1$ and $\tilde{q}>1$ satisfying $\frac{1}{q}+\frac{1}{\tilde{q}}=1$,

$$
\lambda^{\frac{1}{2}+\delta}\left|p^{v}\right| \leq \frac{1}{q} \lambda^{\left(\frac{1}{2}+\delta\right) q}+\frac{1}{\tilde{q}}\left|p^{v}\right|^{\tilde{q}}
$$

follows. Let us take $q=\left(\frac{1}{2}+\delta\right)^{-1}$ for $\delta \in\left(0, \frac{1}{10}\right)$, and hence $\tilde{q}=\left(\frac{1}{2}-\delta\right)^{-1}$. Then we have

$$
\lambda^{\frac{1}{2}+\delta}\left|p^{v}\right| \leq\left(\frac{1}{2}+\delta\right) \lambda+\left(\frac{1}{2}-\delta\right)\left|p^{v}\right|^{\left(\frac{1}{2}-\delta\right)^{-1}} .
$$


Note that

$$
\sup _{\lambda>0, \mathbf{p} \in \mathbf{R}^{d}} \lambda\left(\mathbf{p}^{2}+M^{2}+\lambda\right)^{-1}\left(\sqrt{\mathbf{p}^{2}+M^{2}}+1\right)^{-1 / 2}<\infty .
$$

Since $0<\delta<\frac{1}{10}$, we see that $\left(\frac{1}{2}-\delta\right)^{-1}<\frac{5}{2}$, and hence

$$
\sup _{\mathbf{p}>\mathbf{R}^{d}}\left|p^{v}\right|^{\left(\frac{1}{2}-\delta\right)^{-1}}\left(\mathbf{p}^{2}+M^{2}\right)^{-1}\left(\sqrt{\mathbf{p}^{2}+M^{2}}+1\right)^{-1 / 2}<\infty .
$$

Then we have

$$
\begin{array}{r}
\sup _{\lambda>0, \mathbf{p} \in \mathbf{R}^{d}}\left|p^{v}\right|^{\left(\frac{1}{2}-\delta\right)^{-1}}\left(\mathbf{p}^{2}+M^{2}+\lambda\right)^{-1}\left(\sqrt{p^{2}+M^{2}}+1\right)^{-1 / 2} \\
\quad \leq \sup _{\mathbf{p} \in \mathbf{R}^{d}}\left|p^{\nu}\right|^{\left(\frac{1}{2}-\delta\right)^{-1}}\left(\mathbf{p}^{2}+M^{2}\right)^{-1}\left(\sqrt{\mathbf{p}^{2}+M^{2}}+1\right)^{-1 / 2}<\infty .
\end{array}
$$

By (18), (19) and (21), we obtain (16).

\section{(Proof of Proposition 3.1)}

It follows that for a nonnegative self-adjoint operator $S$,

$$
\sqrt{S} \Phi=\frac{1}{\pi} \int_{0}^{\infty} \frac{1}{\sqrt{\lambda}}(S+\lambda)^{-1} S \Phi d \lambda, \quad \Phi \in \mathcal{D}(S) .
$$

Let

$$
\begin{aligned}
& A_{j}(\Lambda)=\overline{\left(-\triangle_{j} \otimes I+Q_{j}(\Lambda)+M^{2}\right)_{\left\lceil\mathcal{D}_{0}\right.}}, \\
& B_{j}=-\triangle_{j} \otimes I+M^{2} .
\end{aligned}
$$

Then we have for $\Psi \in \mathcal{D}_{0}$,

$$
\begin{aligned}
\left(U(\Lambda)^{-1}\left(H_{\mathrm{p}} \otimes I\right) U(\Lambda)-H_{\mathrm{p}} \otimes I\right) \Psi & =\sum_{j=1}^{N} \frac{1}{\pi} \int_{0}^{\infty} \frac{1}{\sqrt{\lambda}}\left\{\left(A_{j}(\Lambda)+\lambda\right)^{-1} A_{j}(\Lambda)-\left(B_{j}+\lambda\right)^{-1} B_{j}\right\} \Psi d \lambda \\
& =\sum_{j=1}^{N} \frac{1}{\pi} \int_{0}^{\infty} \sqrt{\lambda}\left(A_{j}(\Lambda)+\lambda\right)^{-1}\left(A_{j}(\Lambda)-B_{j}\right)\left(B_{j}+\lambda\right)^{-1} \Psi d \lambda \\
& =\sum_{j=1}^{N} \frac{1}{\pi} \int_{0}^{\infty} \sqrt{\lambda}\left(A_{j}(\Lambda)+\lambda\right)^{-1} Q_{j}(\Lambda)\left(B_{j}+\lambda\right)^{-1} \Psi d \lambda
\end{aligned}
$$

By (13) and the spectral decomposition theorem, $\left\|\left(A_{j}(\Lambda)+\lambda\right)^{-1}\right\| \leq \frac{1}{\lambda+M^{2}}, \lambda>0$ follows, and then we have

$$
\left\|\left(U(\Lambda)^{-1}\left(H_{\mathrm{p}} \otimes I\right) U(\Lambda)-H_{\mathrm{p}} \otimes I\right) \Psi\right\| \leq \sum_{j=1}^{N} \frac{1}{\pi} \int_{0}^{\infty} \frac{\sqrt{\lambda}}{\lambda+M^{2}}\left\|Q_{j}(\Lambda)\left(B_{j}+\lambda\right)^{-1} \Psi\right\| d \lambda .
$$

We see that

$$
\begin{aligned}
\left\|Q_{j}(\Lambda)\left(B_{j}+\lambda\right)^{-1} \Psi\right\| \leq & \left(\frac{\kappa}{\Lambda}\right)\left(\left\|\Pi\left(\frac{\nabla f_{\mathbf{x}_{j}}}{\omega}\right) \cdot\left(\hat{\mathbf{p}_{j}} \otimes I\right)\left(B_{j}+\lambda\right)^{-1} \Psi\right\|+\left\|\Pi\left(\frac{\triangle f_{\mathbf{x}_{j}}}{\omega}\right)\left(B_{j}+\lambda\right)^{-1} \Psi\right\|\right) \\
& +\left(\frac{\kappa}{\Lambda}\right)^{2}\left\|\Pi\left(\frac{\nabla f_{\mathbf{x}_{j}}}{\omega}\right) \cdot \Pi\left(\frac{\nabla f_{\mathbf{x}_{j}}}{\omega}\right)\left(B_{j}+\lambda\right)^{-1} \Psi\right\| .
\end{aligned}
$$


Note that

$$
\begin{aligned}
& \left\|\Pi\left(\frac{\nabla f_{\mathbf{x}_{j}}}{\omega}\right) \cdot\left(\hat{\mathbf{p}_{j}} \otimes I\right)\left(B_{j}+\lambda\right)^{-1} \Psi\right\| \\
& \leq \sum_{V}\left\|\Pi\left(\frac{\partial_{x_{j}^{v}} f_{\mathbf{x}_{j}}}{\omega}\right)\left(I \otimes H_{\mathrm{b}}+1\right)^{-1 / 2}\right\|\left\|\left(\hat{\mathbf{p}}_{j}^{v} \otimes I\right)\left(B_{j}+\lambda\right)^{-1}\left(H_{\mathrm{p}} \otimes I+1\right)^{-1 / 2}\right\|\left\|\left(H_{\mathrm{p}} \otimes I+1\right)^{1 / 2}\left(I \otimes H_{\mathrm{b}}+1\right)^{1 / 2} \Psi\right\|
\end{aligned}
$$

Here we used the boundness (4) and (5). Applying the Lemma 3.2 to $\|\left(\hat{\mathbf{p}}_{j}^{v} \otimes I\right)\left(B_{j}+\lambda\right)^{-1}\left(H_{\mathrm{p}} \otimes I+\right.$ $1)^{-1 / 2} \|$ in (26), it is seen that for $\delta \in\left(0, \frac{1}{10}\right)$, there exist $\alpha_{j}(\delta) \geq 0$ such that

$$
\left\|\Pi\left(\frac{\nabla f_{\mathbf{x}_{j}}}{\omega}\right) \cdot\left(\hat{\mathbf{p}}_{j} \otimes I\right)\left(B_{j}+\lambda\right)^{-1} \Psi\right\| \leq \frac{\alpha_{j}(\delta)}{\lambda \frac{1}{2}+\delta}\left\|\left(H_{\mathrm{p}} \otimes I+1\right)^{1 / 2}\left(I \otimes H_{\mathrm{b}}+1\right)^{1 / 2} \Psi\right\|,
$$

and hence we have

$$
\left\|\Pi\left(\frac{\nabla f_{\mathbf{x}_{j}}}{\omega}\right) \cdot\left(\hat{\mathbf{p}_{j}} \otimes I\right)\left(B_{j}+\lambda\right)^{-1} \Psi\right\| \leq \frac{\alpha_{j}(\delta)}{\lambda^{\frac{1}{2}+\delta}}\left(\left\|H_{\mathrm{p}} \Psi\right\|+\left\|H_{\mathrm{b}} \Psi\right\|+\|\Psi\|\right) .
$$

Since $\left(\left\|H_{\mathrm{p}} \otimes I \Psi\right\|+\left\|I \otimes H_{\mathrm{b}} \Psi\right\|\right)^{2} \leq 2\left\|H_{0}(\Lambda)\right\|^{2}$, we have

$\int_{0}^{\infty} \frac{\sqrt{\lambda}}{\lambda+M^{2}}\left\|\Pi\left(\frac{\nabla f_{\mathbf{x}_{j}}}{\omega}\right) \cdot\left(\hat{\mathbf{p}}_{j} \otimes I\right)\left(B_{j}+\lambda\right)^{-1} \Psi\right\| d \lambda \leq \alpha_{j}(\boldsymbol{\delta})\left(\int_{0}^{\infty} \frac{1}{\left(\lambda+M^{2}\right) \lambda^{\delta}} d \lambda\right)\left(\sqrt{2}\left\|H_{0}(\Lambda) \Psi\right\|+\|\Psi\|\right)$.

By $\left\|\Pi\left(\frac{\triangle f_{\mathbf{x}_{j}}}{\omega}\right)\left(I \otimes H_{\mathrm{b}}+1\right)^{-1 / 2}\right\|<\infty$ and $\left\|\left(B_{j}+\lambda\right)^{-1}\right\| \leq \frac{1}{\lambda+M^{2}}$, we have

$$
\begin{aligned}
\left\|\Pi\left(\frac{\triangle f_{\mathbf{x}_{j}}}{\omega}\right)\left(B_{j}+\lambda\right)^{-1} \Psi\right\| & \leq\left\|\Pi\left(\frac{\triangle f_{\mathbf{x}_{j}}}{\omega}\right)\left(I \otimes H_{\mathrm{b}}+1\right)^{-1 / 2}\right\|\left\|\left(B_{j}+\lambda\right)^{-1}\right\|\left\|\left(I \otimes H_{\mathrm{b}}+1\right)^{1 / 2} \Psi\right\| \\
& \leq \frac{1}{\lambda+M^{2}}\left\|\Pi\left(\frac{\triangle f_{\mathbf{x}_{j}}}{\omega}\right)\left(I \otimes H_{\mathrm{b}}+1\right)^{-1 / 2}\right\|\left\|\left(I \otimes H_{\mathrm{b}}+1\right)^{1 / 2} \Psi\right\| .
\end{aligned}
$$

Then by $\left\|\left(I \otimes H_{\mathrm{b}}+1\right)^{1 / 2} \Psi\right\| \leq\left\|H_{0}(\Lambda) \Psi\right\|+\|\Psi\|$, we have

$$
\begin{aligned}
\int_{0}^{\infty} \frac{\sqrt{\lambda}}{\lambda+M^{2}} \| \Pi\left(\frac{\triangle f_{\mathbf{x}_{j}}}{\omega}\right) & \left(B_{j}+\lambda\right)^{-1} \Psi \| d \lambda \\
& \leq\left\|\Pi\left(\frac{\triangle f_{\mathbf{x}_{j}}}{\omega}\right)\left(I \otimes H_{\mathrm{b}}+1\right)^{-1 / 2}\right\|\left(\int_{0}^{\infty} \frac{\sqrt{\lambda}}{\left(\lambda+M^{2}\right)^{2}} d \lambda\right)\left(\left\|H_{0}(\Lambda) \Psi\right\|+\|\Psi\|\right) .
\end{aligned}
$$

In addition we also see that

$$
\begin{aligned}
\left\|\Pi\left(\frac{\nabla f_{\mathbf{x}_{j}}}{\omega}\right) \cdot \Pi\left(\frac{\nabla f_{\mathbf{x}_{j}}}{\omega}\right)\left(B_{j}+\lambda\right)^{-1} \Psi\right\| & \leq\left\|\Pi\left(\frac{\nabla f_{\mathbf{x}_{j}}}{\omega}\right) \cdot \Pi\left(\frac{\nabla f_{\mathbf{x}_{j}}}{\omega}\right)\left(I \otimes H_{\mathrm{b}}+1\right)^{-1}\right\|\left\|\left(B_{j}+\lambda\right)^{-1}\right\|\left\|\left(I \otimes H_{\mathrm{b}}+1\right) \Psi\right\| \\
& \leq \frac{1}{\lambda+M^{2}}\left\|\Pi\left(\frac{\nabla f_{\mathbf{x}_{j}}}{\omega}\right) \cdot \Pi\left(\frac{\nabla f_{\mathbf{x}_{j}}}{\omega}\right)\left(I \otimes H_{\mathrm{b}}+1\right)^{-1}\right\|\left\|\left(I \otimes H_{\mathrm{b}}+1\right) \Psi\right\| .
\end{aligned}
$$


Since $\left\|\Pi(\xi) \Pi(\eta)\left(H_{\mathrm{b}}+1\right)^{-1}\right\|<\infty$ for $\xi, \eta \in \mathcal{D}(\omega)$, we obtain

$$
\begin{aligned}
& \int_{0}^{\infty} \frac{\sqrt{\lambda}}{\lambda+M^{2}}\left\|\Pi\left(\frac{\nabla f_{\mathbf{x}_{j}}}{\omega}\right) \cdot \Pi\left(\frac{\nabla f_{\mathbf{x}_{j}}}{\omega}\right)\left(B_{j}+\lambda\right)^{-1} \Psi\right\| d \lambda \\
& \quad \leq\left\|\Pi\left(\frac{\nabla f_{\mathbf{x}_{j}}}{\omega}\right) \cdot \Pi\left(\frac{\nabla f_{\mathbf{x}_{j}}}{\omega}\right)\left(H_{\mathrm{b}}+1\right)^{-1}\right\| \|\left(\int_{0}^{\infty} \frac{\sqrt{\lambda}}{\left(\lambda+M^{2}\right)^{2}} d \lambda\right)\left(\left\|H_{0}(\Lambda) \Psi\right\|+\|\Psi\|\right) .
\end{aligned}
$$

Then from (28),(30), (32) and (25), the proposition follows.

Proposition 3.3 Assume (A.1) - (A.4).

(1) For $\varepsilon>0$, there exists $\Lambda(\varepsilon) \geq 0$ such that for all $\Lambda>\Lambda(\varepsilon)$,

$$
\|K(\Lambda) \Psi\| \leq \varepsilon\left\|H_{0}(\Lambda) \Psi\right\|+v(\varepsilon)\|\Psi\|, \quad \Psi \in \mathcal{D}_{0},
$$

holds, where $v(\varepsilon)$ is a constant independent of $\Lambda \geq \Lambda(\varepsilon)$.

(2) Then for all $z \in \mathbf{C} \backslash \mathbf{R}$, it follows that

$$
s-\lim _{\Lambda \rightarrow \infty} K(\Lambda)\left(H_{0}(\Lambda)-z\right)^{-1}=V_{e f f}\left(H_{p}-z\right)^{-1} \otimes P_{\Omega_{b}},
$$

(Proof)

(1) By the condition (A.4), $V_{\text {eff }}$ is bounded. Then (1) follows from Proposition 3.1.

(2) It is seen that

$$
K(\Lambda)\left(H_{0}(\Lambda)-z\right)^{-1}=K(\Lambda)\left(H_{\mathrm{p}}-z\right)^{-1} \otimes P_{\Omega_{\mathrm{b}}}+K(\Lambda)\left(H_{0}(\Lambda)-z\right)^{-1}\left(I \otimes\left(1-P_{\Omega_{\mathrm{b}}}\right)\right) .
$$

By Proposition 3.1, we have

$$
s-\lim _{\Lambda \rightarrow \infty} K(\Lambda)\left(\left(H_{\mathrm{p}}-z\right)^{-1} \otimes P_{\Omega_{\mathrm{b}}}\right) \Psi=V_{\mathrm{eff}}\left(\left(H_{\mathrm{p}}-z\right)^{-1} \otimes P_{\Omega_{\mathrm{b}}}\right) \Psi .
$$

By (33), we see that for $\varepsilon>0$ there exists $\Lambda(\varepsilon) \geq 0$ such that for all $\Lambda>\Lambda(\varepsilon)$

$$
\left\|K(\Lambda)\left(H_{0}(\Lambda)-z\right)^{-1} \Phi\right\| \leq \varepsilon\|\Phi\|+(\varepsilon|z|+v(\varepsilon))\left\|\left(H_{0}(\Lambda)-z\right)^{-1} \Phi\right\|, \quad \Phi \in \mathcal{H} .
$$

Note that $\lim _{\Lambda \rightarrow \infty}\left\|\left(H_{0}(\Lambda)-z\right)^{-1}\left(I \otimes\left(1-P_{\Omega_{\mathrm{b}}}\right)\right) \Psi\right\|=0$, and hence we obtain

$$
\lim _{\Lambda \rightarrow \infty}\left\|K(\Lambda)\left(H_{0}(\Lambda)-z\right)^{-1}\left(I \otimes\left(1-P_{\Omega_{\mathrm{b}}}\right)\right) \Psi\right\|=0 .
$$

By (35) and (36), we obtain (34).

\section{(Proof of Theorem 2.1)}

By Proposition 3.3, it is shown that $H(\Lambda)$ satisfies the condition (S.1) and (S.2) by applying $H_{0}(\Lambda)$ to $A \otimes I+\Lambda I \otimes B$ and $K(\Lambda)$ to $C(\Lambda)$. Hence by the Proposition A, we have for $z \in \mathbf{C} \backslash \mathbf{R}$,

$$
s-\lim _{\Lambda \rightarrow \infty}(H(\Lambda)-z)^{-1}=\lim _{\Lambda \rightarrow \infty} U(\Lambda)\left(H_{0}(\Lambda)-z\right)^{-1} U(\Lambda)^{-1}=\left(H_{\mathrm{p}}+V_{\mathrm{eff}}-z\right)^{-1} \otimes P_{\Omega_{\mathrm{b}}} .
$$

Thus the proof is completed.

\section{Acknowledgments}

It is pleasure to thank Professor Fumio Hiroshima for his advice and comments. 


\section{References}

[1] A. Arai, Asymptotic analysis and its application to the nonrelativistic limit of the Pauli-Fierz and a spinboson model, J. Math. Phys. 32 (1990), 2653-2663.

[2] E. B. Davies, Particle interactions and the weak coupling limit, J. Math. Phys. 20 (1979), 345-351.

[3] I. Daubechies, One electron molecules with relativistic kinetic energy : Properties of the discrete spectrum, Cотти. Math. Phys., 94 (1984), 523-535.

[4] J. Dereziński, The Mourre estimate for dispersive $N$-body Schrödinger operators, Trans. Amer. Math. Soc. 317 (1990), 773-798.

[5] C. Gérard, The mourre estimate for regular dispersive systems, Ann. Inst. H. Poincaré Phys. Théor. 54 (1991), 59-88.

[6] I. Herbst, Spectral theory of the operator $\left(p^{2}+m^{2}\right)^{1 / 2}-Z e^{2} / r$, Commun. Math. Phys., 53 (1977), 285-294.

[7] F. Hiroshima, Analysis of ground states of atoms interacting with a quantized radiation field, Topics in the theory of Shrödinger operators , (H.Araki and H.Ezawa eds. ) World Scientific, 2004, 145-273.

[8] F. Hiroshima, Scaling limit of a model of quantum electrodynamics, J. Math. Phys. 34 (1993), 4478-4518.

[9] F. Hiroshima, Scaling limit of a model of quantum electrodynamics with many nonrelativistic particles, Rev. Math. Phys. 9 (1997), 201-225.

[10] F. Hiroshima, Weak coupling limit with a removal of an ultraviolet cutoff for a Hamiltonian of particles interacting with a massive scalar field, Inf. Dim. Ana. Quantum Prob. Rel. Top. 1 (1998), 407-423.

[11] F. Hiroshima, Weak coupling limit and a removing ultraviolet cutoff for a Hamiltonian of particles interacting with a quantized scalar field, J. Math. Phys. 40 (1999), 1215-1236.

[12] F. Hiroshima, Observable effects and parametrized scaling limits of a model in non-relativistic quantum electrodynamics, J. Math. Phys. 43 (2002), 1755-1795.

[13] F. Hiroshima and H.Spohn, Enhanced binding through coupling to a quantum field, Ann. Henri. Poincaré 2 (2001), 1150-1187.

[14] F. Hiroshima and I.Sasaki, On the ionization of the semi-relativistic Pauli-Fierz model for a single particle, (arxiv : 1003.1661v4).

[15] E. Lieb and M. Loss, Analysis (second edition), Amer. Math. Soc. 2001.

[16] A. Ohkubo, Scaling limit for the Derezinński-Gérard Model, to appear in Hokkaido Math. J.

[17] A. Suzuki, Scaling limits for a general class of quantum field models and its applications to nuclear physics and condensed matter physics, Inf. Dim. Ana. Quantum Prob. Rel. Top. 10 (2007), 43-65.

[18] A. Suzuki, Scaling limits for a generalization of the Nelson model and its application to nuclear physics, Rev. Math. Phys. 19 (2007), 131-155.

[19] T. Takaesu, On the scaling limit of quantum electrodynamics with spatial cutoffs. (arxiv : 0908.2080v1)

[20] T. Umeda, Radiation conditions and resolvent estimates for relativistic Schrödinger operators, Ann. Inst. H. Poincaré Phys. Théor. 63 (1995), 277-296.

[21] R. Weder, Spectral properties of one-body relativistic spin-zero hamiltonians, Ann. Inst. H. Poincaré, Sect. A, 20 (1974), 211-220. 


\section{List of MI Preprint Series, Kyushu University}

The Global COE Program

Math-for-Industry Education \& Research Hub

MI

MI2008-1 Takahiro ITO, Shuichi INOKUCHI \& Yoshihiro MIZOGUCHI

Abstract collision systems simulated by cellular automata

MI2008-2 Eiji ONODERA

The intial value problem for a third-order dispersive flow into compact almost Hermitian manifolds

MI2008-3 Hiroaki KIDO

On isosceles sets in the 4-dimensional Euclidean space

MI2008-4 Hirofumi NOTSU

Numerical computations of cavity flow problems by a pressure stabilized characteristiccurve finite element scheme

MI2008-5 Yoshiyasu OZEKI

Torsion points of abelian varieties with values in nfinite extensions over a padic field

MI2008-6 Yoshiyuki TOMIYAMA

Lifting Galois representations over arbitrary number fields

MI2008-7 Takehiro HIROTSU \& Setsuo TANIGUCHI

The random walk model revisited

MI2008-8 Silvia GANDY, Masaaki KANNO, Hirokazu ANAI \& Kazuhiro YOKOYAMA Optimizing a particular real root of a polynomial by a special cylindrical algebraic decomposition

MI2008-9 Kazufumi KIMOTO, Sho MATSUMOTO \& Masato WAKAYAMA

Alpha-determinant cyclic modules and Jacobi polynomials 
MI2008-10 Sangyeol LEE \& Hiroki MASUDA

Jarque-Bera Normality Test for the Driving Lévy Process of a Discretely Observed Univariate SDE

MI2008-11 Hiroyuki CHIHARA \& Eiji ONODERA

A third order dispersive flow for closed curves into almost Hermitian manifolds

MI2008-12 Takehiko KINOSHITA, Kouji HASHIMOTO and Mitsuhiro T. NAKAO

On the $L^{2}$ a priori error estimates to the finite element solution of elliptic problems with singular adjoint operator

MI2008-13 Jacques FARAUT and Masato WAKAYAMA

Hermitian symmetric spaces of tube type and multivariate Meixner-Pollaczek polynomials

MI2008-14 Takashi NAKAMURA

Riemann zeta-values, Euler polynomials and the best constant of Sobolev inequality

MI2008-15 Takashi NAKAMURA

Some topics related to Hurwitz-Lerch zeta functions

MI2009-1 Yasuhide FUKUMOTO

Global time evolution of viscous vortex rings

MI2009-2 Hidetoshi MATSUI \& Sadanori KONISHI

Regularized functional regression modeling for functional response and predictors

MI2009-3 Hidetoshi MATSUI \& Sadanori KONISHI

Variable selection for functional regression model via the $L_{1}$ regularization

MI2009-4 Shuichi KAWANO \& Sadanori KONISHI

Nonlinear logistic discrimination via regularized Gaussian basis expansions

MI2009-5 Toshiro HIRANOUCHI \& Yuichiro TAGUCHII

Flat modules and Groebner bases over truncated discrete valuation rings 
MI2009-6 Kenji KAJIWARA \& Yasuhiro OHTA

Bilinearization and Casorati determinant solutions to non-autonomous $1+1$ dimensional discrete soliton equations

\section{MI2009-7 Yoshiyuki KAGEI}

Asymptotic behavior of solutions of the compressible Navier-Stokes equation around the plane Couette flow

MI2009-8 Shohei TATEISHI, Hidetoshi MATSUI \& Sadanori KONISHI

Nonlinear regression modeling via the lasso-type regularization

MI2009-9 Takeshi TAKAISHI \& Masato KIMURA

Phase field model for mode III crack growth in two dimensional elasticity

MI2009-10 Shingo SAITO

Generalisation of Mack's formula for claims reserving with arbitrary exponents for the variance assumption

MI2009-11 Kenji KAJIWARA, Masanobu KANEKO, Atsushi NOBE \& Teruhisa TSUDA Ultradiscretization of a solvable two-dimensional chaotic map associated with the Hesse cubic curve

\section{MI2009-12 Tetsu MASUDA}

Hypergeometric T -functions of the q-Painlevé system of type $E_{8}^{(1)}$

MI2009-13 Hidenao IWANE, Hitoshi YANAMI, Hirokazu ANAI \& Kazuhiro YOKOYAMA A Practical Implementation of a Symbolic-Numeric Cylindrical Algebraic Decomposition for Quantifier Elimination

MI2009-14 Yasunori MAEKAWA

On Gaussian decay estimates of solutions to some linear elliptic equations and its applications

MI2009-15 Yuya ISHIHARA \& Yoshiyuki KAGEI

Large time behavior of the semigroup on $L^{p}$ spaces associated with the linearized compressible Navier-Stokes equation in a cylindrical domain 
MI2009-16 Chikashi ARITA, Atsuo KUNIBA, Kazumitsu SAKAI \& Tsuyoshi SAWABE Spectrum in multi-species asymmetric simple exclusion process on a ring

MI2009-17 Masato WAKAYAMA \& Keitaro YAMAMOTO

Non-linear algebraic differential equations satisfied by certain family of elliptic functions

MI2009-18 Me Me NAING \& Yasuhide FUKUMOTO

Local Instability of an Elliptical Flow Subjected to a Coriolis Force

MI2009-19 Mitsunori KAYANO \& Sadanori KONISHI

Sparse functional principal component analysis via regularized basis expansions and its application

MI2009-20 Shuichi KAWANO \& Sadanori KONISHI

Semi-supervised logistic discrimination via regularized Gaussian basis expansions

MI2009-21 Hiroshi YOSHIDA, Yoshihiro MIWA \& Masanobu KANEKO

Elliptic curves and Fibonacci numbers arising from Lindenmayer system with symbolic computations

MI2009-22 Eiji ONODERA

A remark on the global existence of a third order dispersive flow into locally Hermitian symmetric spaces

MI2009-23 Stjepan LUGOMER \& Yasuhide FUKUMOTO

Generation of ribbons, helicoids and complex scherk surface in laser-matter Interactions

MI2009-24 Yu KAWAKAMI

Recent progress in value distribution of the hyperbolic Gauss map

MI2009-25 Takehiko KINOSHITA \& Mitsuhiro T. NAKAO

On very accurate enclosure of the optimal constant in the a priori error estimates for $H_{0}^{2}$-projection 
MI2009-26 Manabu YOSHIDA

Ramification of local fields and Fontaine's property (Pm)

MI2009-27 Yu KAWAKAMI

Value distribution of the hyperbolic Gauss maps for flat fronts in hyperbolic three-space

MI2009-28 Masahisa TABATA

Numerical simulation of fluid movement in an hourglass by an energy-stable finite element scheme

MI2009-29 Yoshiyuki KAGEI \& Yasunori MAEKAWA Asymptotic behaviors of solutions to evolution equations in the presence of translation and scaling invariance

MI2009-30 Yoshiyuki KAGEI \& Yasunori MAEKAWA

On asymptotic behaviors of solutions to parabolic systems modelling chemotaxis

MI2009-31 Masato WAKAYAMA \& Yoshinori YAMASAKI

Hecke's zeros and higher depth determinants

MI2009-32 Olivier PIRONNEAU \& Masahisa TABATA

Stability and convergence of a Galerkin-characteristics finite element scheme of lumped mass type

MI2009-33 Chikashi ARITA

Queueing process with excluded-volume effect

MI2009-34 Kenji KAJIWARA, Nobutaka NAKAZONO \& Teruhisa TSUDA

Projective reduction of the discrete Painlevé system of type $\left(A_{2}+A_{1}\right)^{(1)}$

MI2009-35 Yosuke MIZUYAMA, Takamasa SHINDE, Masahisa TABATA \& Daisuke TAGAMI Finite element computation for scattering problems of micro-hologram using DtN map 
MI2009-36 Reiichiro KAWAI \& Hiroki MASUDA

Exact simulation of finite variation tempered stable Ornstein-Uhlenbeck processes

MI2009-37 Hiroki MASUDA

On statistical aspects in calibrating a geometric skewed stable asset price model

MI2010-1 Hiroki MASUDA

Approximate self-weighted LAD estimation of discretely observed ergodic OrnsteinUhlenbeck processes

MI2010-2 Reiichiro KAWAI \& Hiroki MASUDA

Infinite variation tempered stable Ornstein-Uhlenbeck processes with discrete observations

MI2010-3 Kei HIROSE, Shuichi KAWANO, Daisuke MIIKE \& Sadanori KONISHI Hyper-parameter selection in Bayesian structural equation models

MI2010-4 Nobuyuki IKEDA \& Setsuo TANIGUCHI

The Itô-Nisio theorem, quadratic Wiener functionals, and 1-solitons

MI2010-5 Shohei TATEISHI \& Sadanori KONISHI

Nonlinear regression modeling and detecting change point via the relevance vector machine

MI2010-6 Shuichi KAWANO, Toshihiro MISUMI \& Sadanori KONISHI

Semi-supervised logistic discrimination via graph-based regularization

MI2010-7 Teruhisa TSUDA

UC hierarchy and monodromy preserving deformation

MI2010-8 Takahiro ITO

Abstract collision systems on groups

MI2010-9 Hiroshi YOSHIDA, Kinji KIMURA, Naoki YOSHIDA, Junko TANAKA \& Yoshihiro MIWA

An algebraic approach to underdetermined experiments 
MI2010-10 Kei HIROSE \& Sadanori KONISHI

Variable selection via the grouped weighted lasso for factor analysis models

MI2010-11 Katsusuke NABESHIMA \& Hiroshi YOSHIDA

Derivation of specific conditions with Comprehensive Groebner Systems

MI2010-12 Yoshiyuki KAGEI, Yu NAGAFUCHI \& Takeshi SUDOU

Decay estimates on solutions of the linearized compressible Navier-Stokes equation around a Poiseuille type flow

MI2010-13 Reiichiro KAWAI \& Hiroki MASUDA

On simulation of tempered stable random variates

MI2010-14 Yoshiyasu OZEKI

Non-existence of certain Galois representations with a uniform tame inertia weight

MI2010-15 Me Me NAING \& Yasuhide FUKUMOTO

Local Instability of a Rotating Flow Driven by Precession of Arbitrary Frequency

MI2010-16 Yu KAWAKAMI \& Daisuke NAKAJO

The value distribution of the Gauss map of improper affine spheres

MI2010-17 Kazunori YASUTAKE

On the classification of rank 2 almost Fano bundles on projective space

MI2010-18 Toshimitsu TAKAESU

Scaling limits for the system of semi-relativistic particles coupled to a scalar bose field 